\title{
An Exercise in Technology Prioritization in a Competitive Environment
}

\author{
Karen L. Stephens* \\ NASA George C. Marshall Space Flight Center, AL 35812
}

\begin{abstract}
The proper prioritization of technologies within a technology development program is critical for success in a budget constrained environment. A large portfolio of diverse products at differing states of development all competing for program resources presents a need for a process by which a realistic need date can guide the development strategy for each technology. This paper will document an exercise to identify "mission pull" for each technology that can be used to identify the best projected need date for each. This date is then used to back out a development schedule with corresponding funding profile needed to meet the flight opportunity.
\end{abstract}

\section{Introduction}

"Fly anytime, anywhere and complete a host of science objectives at the destination" is a far-reaching goal in the development of advanced in-space technologies for future science and exploration missions. Reduced trip times, reduced reliance on planetary assists, superior maneuverability and smaller launch vehicles can reduce cost and enable greater science return. ${ }^{1}$ Development of advanced in-space propulsion technologies is the charter of the Science Mission Directorate's (SMD) In-Space Propulsion Technology (ISPT) Program managed at the Marshall Space Flight Center. The ISPT Program includes Solar Electric, Aerocapture, Solar Sail, and Advanced Chemical propulsion technologies applicable to a diverse set of interplanetary missions. ISPT products will improve performance over existing systems and thereby enhance existing mission plans and enable previously infeasible missions. Managing this diverse set of technologies is a challenge and optimal allocation of available resources is a chief concern.

\section{Description and Status of ISP Technologies}

The ISPT portfolio includes multiple products within the four technology areas that are advancing propulsion system capability resulting in new materials, manufacturing processes, sub-scale test articles and full-scale engineering system models ${ }^{2}$.

Solar Electric Propulsion is one of the four propulsion technology areas in development. SEP systems require less propellant at launch and on-orbit than chemical systems and are capable of generating low thrust over long periods of time, accelerating a vehicle to velocities higher than that achievable with chemical systems. Time is saved without the need to schedule the mission for launch window or extend for gravity-assists. Advances in SEP include the development of a prototype model 40-cm NASA Evolutionary Xenon Thruster (NEXT) with prototype power processing unit and propellant feed system for integration and system test to $7 \mathrm{~kW}$. ISTP completed long-life testing of the Deep Space 1 (DS-1) NSTAR engine to $283 \%$ of its design life. A High Voltage Hall Accelerator (HiVHAC) thruster has been fabricated and will undergo performance testing this summer.

An Aerocapture vehicle is "captured" into orbit as it makes a single pass through the atmosphere of a planetary body, without the use of on-board propulsion. Studies have shown this "fuel free" method could reduce the typical mass of missions to Mars, Titan, Venus, Saturn and Neptune by $40-80 \%$, allowing more mass for science, a smaller, less expensive launch vehicle or much shorter trip times without the need for time-consuming gravity assists. Recent progress by ISPT includes the design and development of a 2-meter diameter carbon-carbon monocoque areoshell for non-destructive testing, arc-jet testing of ablative and non-ablative TPS materials with integrated heat flux and recession sensors, and improved, light-weight aeroshell structures. ${ }^{2}$ Continued development of both blunt body and lifting body aeroshell configurations as well as initial development of inflatables is planned.

\footnotetext{
${ }^{*}$ Assistant Manager, ISPT Systems Analysis, VP51, MSFC AL 35812, AIAA Member.
} 
Solar Sails provide "propellantless" propulsion using photons, or sunlight, to propel a large, reflective sail that needs no engine or fuel. The thrust continues indefinitely, extending the duration of candidate missions and providing payload mass for science. ISPT has been focusing on three-axis stabilized, square Solar Sails. Two industry teams have fabricated and tested 10 and 20 -meter sub-scale sails with competing approaches, one of an inflatable, rigidizable boom configuration using articulated vanes in at the corners of the square for control and the other a coilable longeron boom system with sliding ballast masses to offset the center mass from the center of pressure of the sail. The development of the $14 \mathrm{~g} / \mathrm{m}^{2}$ sail and pursuit of a lighter-weight $3-7 \mathrm{~g} / \mathrm{m}^{2}$ sail is planned ${ }^{2}$.

Advanced Chemical propulsion is pursuing incremental improvements in state-of-the-art chemical propulsion systems, including improvements to bi-propellant and pump-fed systems, and development in materials and manufacturing processes and thermal design, the latter to pave the way for high-temperature thrusters for higher performance engines ${ }^{2}$.

Much has been accomplished, but new technologies need to be flight demonstrated. Aerocapture and Solar Sail technologies have been selected to compete for the New Millennium Program ST-9 technology demonstration mission. Task flows through the development cycle to a first flight opportunity for all products have been developed but funding must be carefully prioritized to maintain an overall project flow that is strategically timed to bring propulsion solutions to formulators as the missions are being defined by NASA mission developers or PI-lead teams.

\section{The Importance of Optimal Resource Allocation}

How does a program properly allocate resources for competing technology developments? Equal funding for all, the loudest technology manager, the technology most recently successful, the last person to present, are all possible strategies for commanding development funding. These might suffice in times of healthy budgets but decision makers must make tough choices when resources are limited.

The technology development process provides a methodical approach to the problem. A top-down productfocused technology development process begins with a mission need which decomposes into systems needs and then into technology needs with associated requirements ${ }^{3}$. In preparation for a NASA Research Announcement scheduled for early '06 and expecting a reduction in funding within the Science Mission Directorate, the ISPT management team conceived a plan to reassess the missions currently targeted for each technology, identify the current "mission need" and use any changes to reassess program funding priorities.

\section{Historical Data for Applicability Assessments}

A NASA-wide team performed an initial activity to identify and prioritize candidate advanced propulsion technologies for high-priority SMD missions. The report of that process in the Integrated In-Space Transportation Plan (IISTP) recommended the process be repeated bi-annually to keep up with evolving agency focus ${ }^{1}$. Each year since then, a study performed by the ISTP has provided this recommended reassessment.

The IISTP was the original study convening 100 technologists and mission specialists across the agency to assess a broad set of new propulsion technologies that were strong candidates for ISPT funding. A diverse set of missions of interest to the SMD were listed and nine selected as the most comprehensively challenging set that could be analyzed in the study timeframe. Mission experts referred to previous studies or performed new analysis to form the basis for discussion in which a "score" was generated for each technology for each mission. Scores for each technology were added together with the highest scores revealing the most applicable technologies for the broadest mission capture.

The Space Transportation Element Applicability Matrix (STEAM) was performed originally in FY03 with an update and enhancement (STEAM II) in FY04. They were performed for the NASA Space Architect by a panel of mission and propulsion experts from across the agency to assess applicability for 18 technologies to a comprehensive set of 51 missions, both Robotic and Human, from LEO to beyond the solar system. This included many possible mission architectures to capture data for as many missions as was feasible for the study period. Each technology was assessed for each mission and a color code assigned, from green which was "highly applicable" to red which was "not applicable". Discussion for each case was condensed into comments to support the color code assigned. The result was a large database of mission information and technology assessments which produced lists 
of applicable missions for each technology with supporting data. A histogram of the additive results of applicable missions revealed the technologies with the most mission capture in each mission category for each Regime and across all categories.

The Capabilities, Requirements and Applicability Index (CRAI) activity began in 2004 with the in-space propulsion topic covering SEP, Aerocapture and Solar Sails as well as other technologies related to in-space activities other than primary propulsion. The assessment for each technology was the current TRL, technology gaps and applicable missions. The product was a roadmap for each, outlining technology development activities supporting mission readiness for which the technology was assessed as "enabling" or "enhancing".

The most recent references were the newly revised, draft Solar System Exploration (SSE) and Sun Solar System Connection (SSSE) Strategic Roadmaps (May 2005). These are the most recent plans to implement the President's Vision for Space Exploration initiative. They include recommendations from the National Research Council (NRC) Decadal Survey, guiding principles followed, and current plans for NASA's research programs. The SSE mentions Solar Electric, Aerocapture and Advanced Chemical technologies as key technology developments for the roadmap. The SSSC specifies Solar Sails as pivotal in plans for the future.

All previous activities provided historical data applicable directly or by similarity, helping a small team of past participants generate assessments for the updated mission list in a brief period of time. The databases captured expert opinion that was debated for a consensus view and presented to SMD leadership for review.

\section{Development of the Mission List and Assessment Matrix}

Mission definitions evolve over time, new missions are conceived by mission planners and agency priorities change. Though each previous activity generated a mission list, the current SSE and SSSC roadmaps provided the latest priorities for SMD missions and was the source for a new mission list that contained some perennial and evolved versions of earlier concepts as well as inclusion of missions selected competitively in the Discovery Program and New Frontiers Programs.

The New Frontiers missions are competed but are guided by the National Research Council (NRC) Report, "New Frontiers in the Solar System- An Integrated Exploration Strategy" which identified five high priority missions. These were included in the mission list and assessed for applicability.

Because the Discovery class missions are not known until they are awarded, the team consulted past studies and the technical area managers (TAM)s to provide insight on small class missions to which their technology is both applicable and affordable. The SSE roadmap notes the goal of the Discovery program is to start a new mission every two years. The most recent Discovery AO release was January 2006 and is projected every two years for the next decade which allows estimation of upcoming Discovery mission opportunities. The New Frontiers program has a goal to fly three missions in the next decade. A New Frontiers AO is expected in late 2006 which would put the next two in 2010 and 2013 approximately. The anticipated AO dates for both programs became possible targets. The TAM could make the case that one of these would be his "mission pull" based on study results and sufficient technology maturity at a time to begin a proposal.

There were a total of 30 missions that ISPT technologies were thought to be applicable for, 10 noted in the SSE and 20 in the SSSC roadmap. The SSSC provided a flow for the Solar Sails Missions from technology development to the most challenging and costly Interstellar Probe. The class for each mission was noted, Discovery, New Frontiers and Flagship classes of missions (which have cost ranges from $\$ 300-\$ 500 \mathrm{M}, \$ 500-800 \mathrm{M}$ and $\$ 800$ and above, respectively). A projected launch date and additional information to describe the mission was added to the mission list database. 


\begin{tabular}{|l|l|c|}
\hline \multicolumn{1}{|c|}{ Mission Class } & \multicolumn{1}{|c|}{ Mission Title } & $\begin{array}{c}\text { Est Launch } \\
\text { Date }\end{array}$ \\
\hline Discovery & TBD-open competition, not guided by NRC & Every 2 yrs \\
\hline New Frontiers & Pluto-Kuiper Belt Explorer & 2006 \\
\hline New Frontiers & Lunar South Pole Aitken Basin & 2010 \\
\hline New Frontiers & Jupiter Polar Orbiter with Probes & 2010 \\
\hline New Frontiers & Venus In-Situ Explorer & 2013 \\
\hline New Frontiers & Comet Surface (Nucleus) Sample Return (CSSR) & 2013 \\
\hline Flagship & Titan Explorer & 2016 \\
\hline Flagship & Neptune System Mission & 2018 \\
\hline Flagship & Comet Cryo Nucleus Sample Return & 2020 \\
\hline Flagship & Venus Sample Return & 2020 \\
\hline Flagship & Europa Astrobiology Lander & 2025 \\
\hline Living with a Star & Heliostorm & 2016 \\
\hline Living with a Star & Solar Polar Imager & 2026 \\
\hline Flagship & Interstellar Probe & 2035 \\
\hline
\end{tabular}

Table 1 List of missions from SSE and SSSC Roadmaps

The list of missions was then used to generate a matrix of missions and ISPT technologies including first and second generation products. This matrix was the tool used for capturing the assessments.

A team of experts and participants in the past studies met to perform the assessments. A ground rule for the effort was that missions easily accomplished using state-of-the-art chemical propulsion would be applicable but not considered to provide a "mission pull". Only those missions for which ISPT technologies were thought to be enabling or enhancing were rated.

One difficulty in estimating the usefulness of a technology for a mission is that each mission can be performed many different ways with differing objectives. It is important to identify the mission objective and the current thinking on the best way to accomplish the mission goals in order to correctly appraise applicability. Mission length constraints, program cost cap, etc., all affect the mission and the possible need for new technology. The IISTP report contained a detailed mission description for the 9 missions assessed. The STEAM included a brief mission description within the matrix for each of the 51 missions assessed. The SSSE roadmap specified several missions as distinctly Solar Sail missions and included mission descriptions for all missions. In cases where there was little definition, a best guess was made based on current knowledge of SMD objectives and the similarity to more defined missions.

The completion of the matrix involved discussion and reference to past studies with a resulting consensus of the applicability of each technology for each mission. A rating was made for applicable to highly applicable/enhancing/enabling. The nearer the possible launch date, the greater the "mission pull" making it a nearterm, high priority.

\section{Determining the Mission Need Date}

Missions are typically broken into phases for planning purposes. Flight missions progress from award to Concept Study (Phase A), Preliminary Design (Phase B), Final Design and development of Flight Hardware, Spacecraft Bus and Mission Operations Software and Equipment (Phase C), Spacecraft Assembly, Test and Launch Operations (Phase D), and Mission Operations and Data Analysis (Phase E) ${ }^{4}$. It was a finding in the CRAI activity and the consensus of the team that in light of the current environment of limited budgets and risk aversion, a mission including a new technology could be compelling enough to be selected for Phase A study but would be seen as too high risk (without a flight demonstration), to prevail in down-selection over a demonstrated technology. Additionally, with Phase B beginning the mission design, technology development would have to give way for the design phase. This set the cut-off for technology development and flight demonstration at the end Phase A.

The beginning of Phase B could be backed out from a launch date or projected forward from an AO date if the length of each of the phases was known. AO guidelines dictate the length of Phase A which is roughly one year including three months for proposal development, 3 months for selection and 6-9 months for the mission concept 
study. Discovery/New Frontiers, New Millennium Program and European Space Agency missions were examined for a historical estimate of the length of Phase B, C and D. Phase B was approximately 1.5 years for all but Phase $\mathrm{C} / \mathrm{D}$ increased for increasing mission cost. Phase $\mathrm{C} / \mathrm{D}$ was roughly 3.5 years for a small mission $(\angle \$ 300 \mathrm{M}), 4$ years for a medium $(\$ 300 \mathrm{M}-\$ 750 \mathrm{M}), 5$ years for an intermediate $(\$ 750 \mathrm{M}-\$ 1.2 \mathrm{~B})$ and 6 years for a larger mission $(>\$ 1.2 B)^{4}$. This information was the basis of a template for all classes of missions.

The next step was to lay out this information in a manner that provide a visual flow of the mission phases based on the AO date or mission launch date. A Gantt Chart is a graphical technique that is a well known project management tool. It is a planning method that shows the start, milestones and time necessary for completion along a timeline ${ }^{5}$.

A Gantt chart was developed using the mission phases template for each of the missions identified as the earliest "mission pull" for each of the technologies. The Gantt chart showed graphically the phases of the target missions and most importantly, projected need date for each technology based on the beginning of Phase B, which was agreed to be the "need date" for that technology. This was presented to the TAM and ISTP management for review and concurrence. In some cases, the TAM was able to see that their efforts would need to increase to meet the need date. In others, it was apparent that the team could slow down development and still meet the need date. Further discussion included efforts to project interim products for earlier demonstration that could apply to earlier missions and support the need to continue funding as planned. The final result was a funding plan that closely matched the need for each technology to meet it's identified "mission pull" which freed some resources for earlier targets and post-poned development for technologies needed by later missions.

\section{Acknowledgments}

The work described in this paper was funded in whole or in part by the In-Space Propulsion Technology Program, which is managed by NASA's Science Mission Directorate in Washington, D.C., and implemented by the In-Space Propulsion Technology Office at Marshall Space Flight Center in Huntsville, Ala. The program objective is to develop in-space propulsion technologies that can enable or benefit near and mid-term NASA space science missions by significantly reducing cost, mass or travel times. .

\section{References}

${ }^{1}$ Farris, B., Eberle, B., Woodcock, G., and Negast, B., "Integrated In-Space Transportation Plan," NASA/CR-2002-212050,

${ }^{2}$ Johnson, L., Meyer, R.A., Frame, K., "In-Space Propulsion Technologies for Robotic Exploration of the Solar System," AIAA (unpublished).

${ }^{3}$ D. Stanley, "Technology Engineering: the Concurrent Development of Space Transportation Systems and Technology", AIAA-2003-6359, 2003

4 "The Solar System Exploration Strategic Roadmap" and the "Sun-Solar System Connection Strategic Connection", Science Mission Directorate (draft 2005).

${ }_{5}$ M. Largent, S. Mavris "Formulation of a Process for the Planning and Management of Technology Development", AIAA2001-5258, 2001 